\title{
CARACTERIZAÇÃO E ANÁLISE ESPACIAL DO DÉFICIT DE ARMAZENAGEM EM MINAS GERAIS
}

\author{
Marlon Fernandes de Souza ${ }^{1}$ \\ Carlos Fernando Ferreira Lobo ${ }^{2}$
}

\section{Resumo}

O armazenamento de grão é fundamental para o equilíbrio da economia e garantia da segurança alimentar. Em todo o Brasil, a produção de grãos tem alcançado sucessivos recordes, sem que haja investimentos na mesma proporção na pós-colheita. A carência de estruturas para armazenamento da produção pode gerar perdas significativas. Minas Gerais sempre foi um estado importante na produção agrícola brasileira, e apresenta um déficit atual de 6,5 milhões de toneladas de capacidade de armazenamento (CONAB, 2016; IBGE, 2016a). A pesquisa analisou, através da regressão espacial, a relação entre produção e armazenamento de grãos, identificando as áreas do estado de Minas Gerais que apresentam os maiores desvios, com especial atenção ao déficit de armazenagem. A análise de regressão espacial permite modelar, examinar e explorar relacionamentos espaciais e pode ajudar a explicar os fatores por trás dos padrões espaciais observados. O método adotado foi a Regressão Geograficamente Ponderada. A regressão obteve $\mathrm{R}^{2}$ de 0,687 , apesar da grande dispersão dos dados observados e dos elevados valores de resíduos da regressão. Os municípios com maior desvio de armazenagem esperada de acordo com nível de produção, em geral tem seus desvios justificados por outras variáveis que não foram consideradas no modelo.

Palavras-chave:déficit de armazenagem, GWR, estatística especial, pós-colheita, logística.

\begin{abstract}
Grain storage is fundamental to balancing the economy and ensuring food security. Throughout Brazil, grain production has reached successive records, without investments in the same proportion in the post-harvest. The lack of structures to store production can generate significant losses. Minas Gerais has always been an important state in Brazilian agricultural production, with a current deficit of 6.5 million tons of storage capacity (CONAB, 2016; IBGE, 2016a). The research analyzed, through spatial regression, the relationship between production and storage of grains, identifying the areas of the state of Minas Gerais that present the greatest deviations, with special attention to the storage deficit. Spatial regression analysis allows modeling, examining, and exploring spatial relationships and can help explain the factors behind spatial patterns observed. The method adopted was the Geographically Weighted Regression. The regression obtained $\mathrm{R}^{2}$ of 0.687 , despite the great dispersion of the observed data and high regression residuals. The municipalities with the largest expected storage deviation according to the level of production, usually have their deviations justified by other variables that were not considered in the model.
\end{abstract}

key-words:storage deficit, GWR, spatial statistics, post-harvest, logistics.

${ }^{1}$ UFMG/IGC (Mestrando em Análise e Modelagem de Sist. Ambientais) marlonfsouza@ufmg.br

${ }^{2}$ UFMG/IGC (Doutor em Geografia) carlosfflobo@gmail.com 


\section{1- INTRODUÇÃO}

A armazenagem de grãos é fundamental para equalizar oferta e demanda,possibilitando que uma demanda contínua de produtos seja suprida por uma oferta sazonal e sujeita a oscilações, definida pelas safras. Deste modo, o armazenamento é de suma importância para o equilíbrio da economia e garantia da segurança alimentar da população.

Com um notável crescimento nos últimos anos, a produção agrícola éindubitavelmente importante para o Brasil, sendo responsável por 13,94\% do PIB (CEPEA, 2017). Contudo, os sistemas de armazenagem não acompanharam este crescimento. Apesar do aumento da capacidade estática, fruto dos programas de fomento3 desenvolvidos pelo governo e dos grandes investimentos realizados pela iniciativa privada, existe um grande déficit de armazenagem em praticamente todas as regiões do Brasil.

Tão importante quanto o investimento na construção de novas unidades, queaumentarão a capacidade estática de armazenamento, é o posicionamento adequado dessas novas instalações. Pois um armazém bem localizado na cadeia logística reduz custos e agrega valor, enquanto um armazém mal posicionado onera a cadeia, devido ao alto investimento e elevados custos de manutenção.

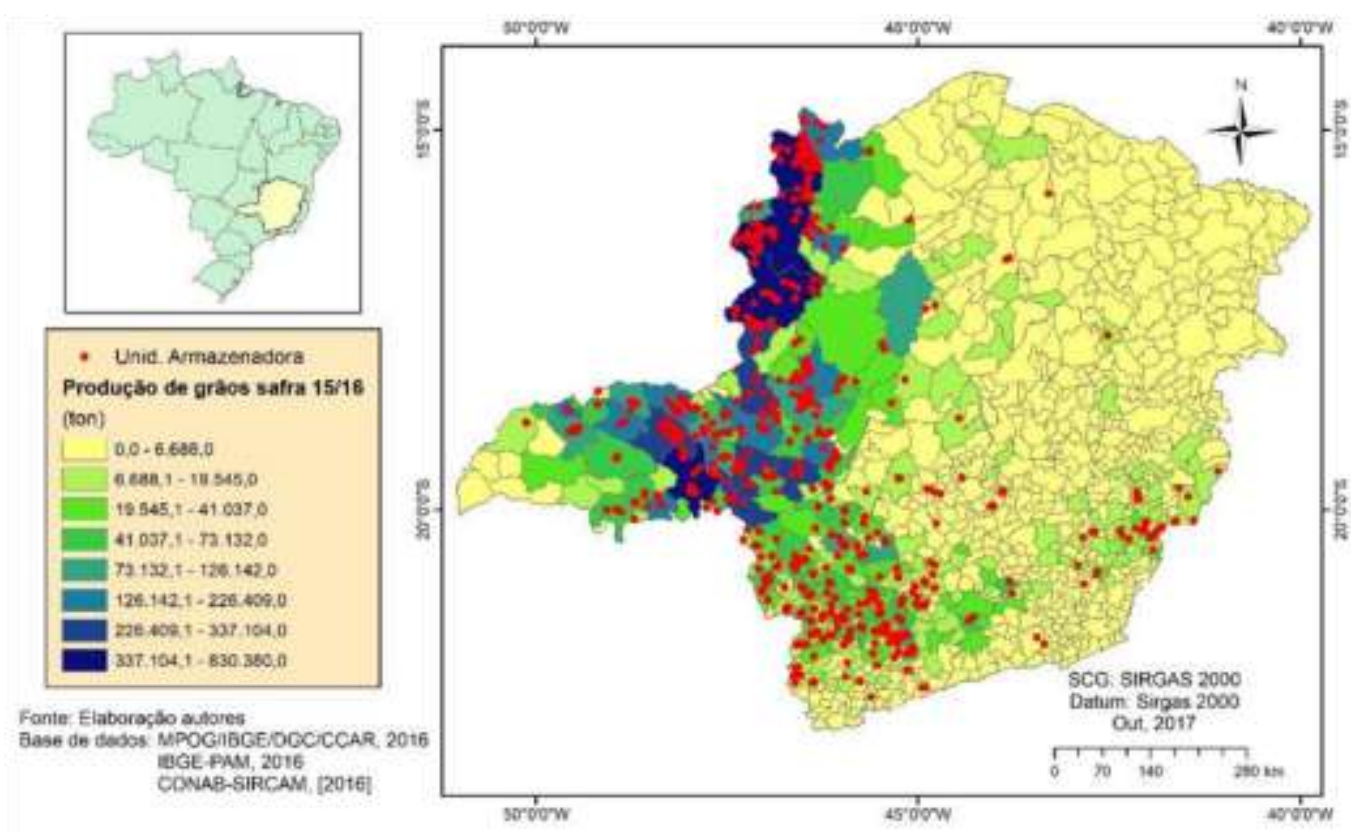

Figura 1. Distribuição da produção de cereais, leguminosas, oleaginosas e café e a localização das unidades armazenadoras de Minas Gerais ${ }^{4}$.

${ }^{3}$ PRONAF; PRONAMP; FINAME Agrícola; MODERAGRO. Além dos atuais e mais importantes: INOVAGRO; PCA e MODERINFRA.

${ }^{4}$ Cadastradas no Sistema de Cadastro Nacional de Unidades Armazenadoras - SICARM/CONAB. 
Existe uma dependência lógica entre a capacidade estática de armazenamento e aprodução de grãos como pode ser observado na Figura 1. Poderíamos dizer que a capacidade estática de armazenamento é uma variável dependente da produção, pois só é interessante a construção de uma unidade armazenadora onde a produção seja suficientemente grande para gerar excedente armazenável. O objetivo desta pesquisa é analisar, através da regressão espacial, a relação entre produção e armazenamento de grãos, identificando as áreas do estado de Minas Gerais que apresentam os maiores resíduos padronizados, que representam déficit (desvios negativos) ou excedente (desvios positivos) de armazenagem.

\section{2- REVISÃO BIBLIOGRÁFICA}

Biageet al. (2002) determina funções intrínsecas e extrínsecas ligadas diretamentea armazenagem de grãos. As funções intrínsecas correspondem à conservação da qualidade, controle de perdas e estocagem de excedente. As funções extrínsecas estão relacionadas com a logística de produção, logística de transporte, suporte a comercialização, estoques reguladores, e auxílio as políticas de segurança alimentar.

Considerando a recomendação da FAO para que a capacidade estática dearmazenamento seja pelo menos 20\% superior a produção de grãos (FAO, 2011 apud PATINO et al., 2013). Minas apresenta um déficit de 6,5 milhões de toneladas de capacidade de armazenamento, pois possui 9.528.286 t de capacidade estática (CONAB, 2016) perante uma produção de 13.364.105 t (IBGE, 2016a). Nos últimos 15 anos, Minas apresentou taxa média de crescimento da capacidade estática de 4,8\%. Com relação ao tipo de armazenagem, o estado se destaca por possuir 3,1 milhões de capacidade do tipo convencional, utilizada basicamente para armazenamento de café (CONAB, 2017a).

A regressão é uma ferramenta estatística que avalia a relação entre duas ou maisvariáveis, tal que uma variável possa ser explicada pela outra. Matematicamente essa relação é dada pela Equação 1 a seguir.

$$
Y=a_{1} X_{1}+a_{2} X_{2}+a_{3} X_{3}+\cdots+a_{\mathrm{n}} X_{\mathrm{n}}+b+\varepsilon
$$

Onde:

$\mathrm{Y}$ : variável dependente

$\mathrm{X}_{1}, \mathrm{X}_{2}, \mathrm{X}_{3}, \ldots, \mathrm{X}_{\mathrm{n}}$ : variáveis explicativas

$a_{1}, a_{2}, a_{3}, \ldots, a_{n}:$ coeficientes da

regressão b: coeficiente de intercepto $\varepsilon$ :

erro aleatório 
O uso de modelos de regressão espacial que incorporam a questão da dependênciaespacial dos dados é recente e apresenta resultados promissores, pois permite a incorporação da problemática ${ }^{5}$ da autocorrelação espacial existente neste tipo de estudo (OVERMARS et al., 2003, SOARES FILHO et al., 2008).

Os modelos de regressão espacial podem ser globais ou locais. Os modelos globaisincluem um parâmetro para capturar a estrutura de auto correlação espacial na área de estudo como um todo. Partem do pressuposto que o processo espacial analisado é estacionário e pode ser capturado em um único parâmetro. Nos modelos de regressão locais os parâmetros variam continuamente no espaço. Sendo divididos em modelos de regressão com efeitos espaciais discretos e modelos com efeitos espaciais contínuos (MITCHELL, 2005).

A Regressão Geograficamente Ponderada (em inglês GeographicallyWeightedRegression - GWR) é um modelo que considera os efeitos espaciais contínuos. A técnica proposta por Brunsdon, Fotheringham e Charlton (1996), é utilizada para modelar processos heterogêneos não estacionários espacialmente, isto é, processos que variam - seja na média, mediana, variância etc. de região para região. A técnica tenta capturar essa variação calibrando um modelo de regressão múltipla que permite que diferentes relações existam em diferentes pontos do espaço. A ideia é vagamente baseada na análise de kernel. A aplicação da GWR pode ser observada em diferentes áreas de pesquisa, tais como Geografia (LOBO et al., 2015; SEE et al., 2015), Saúde (GILBERT e CHAKRABORTY, 2011), Ciências Agrárias (SHARMA et al., 2011), Transportes (CARVALHO, 2006; MENDONÇA, 2008) e Economia e Finanças (ALBUQUERQUE et al., 2017; HUANG e LEUNG, 2002).

\section{3- MATERIAIS E MÉTODOS}

\section{1- Caracterizações da área de estudos}

Minas Gerais é o quarto maior estado do Brasil, com área territorial de 586.520,732km² (IBGE, 2016c) e o segundo em quantidade de habitantes, 21.119.536 (IBGE, 2017), localizado na região Sudeste do país. Seu território é subdividido em 853 municípios, a maior quantidade dentre os estados brasileiros.

\footnotetext{
${ }^{5}$ Quando se constata autocorrelação espacial, a partir da análise dos resíduos, quebra-se o pressuposto de independência entre amostras necessária para utilização da regressão clássica. Caso prossiga-se com a análise, os resultados estatísticos podem ser influenciados, apresentando vinculação estatística onde não existe. A alternativa então, é a utilização de modelos de regressão espacial. Uma das premissas para essa regressão é a admissão de uma estrutura de dependência espacial.
} 
Detentor do terceiro maior PIB do país, R 519.326 milhões em 2015 (FJP, 2017a). Minas tem acumulado sucessivas variações negativas em volume em relação ao ano anterior, 0,7\% em 2014, $-4,3 \%$ em 2015 e $-2,6 \%{ }^{6}$ no ano de 2016. Além de reduzir a participação no PIB nacional ao longo da última década. Em 2016, o setor agropecuário foi o responsável por atenuar a queda da atividade econômica em comparação com a economia nacional. O resultado positivo do PIB agropecuário mineiro $(6,6 \%)$ se deve a robusta expansão na quantidade produzida de café e soja, os dois principais produtos da pauta agrícola (FJP, 2017b).

Sempre importante na produção agrícola brasileira, Minas possui o quinto maiorvalor bruto da produção (VBP) agrícola, avaliado em R \$33,9 bilhões (MAPA, 2017a). Contudo o crescimento significativo da produção de grãos ao longo dos últimos anos não foi acompanhado pela armazenagem, como pode ser observado no Gráfico 1.

Gráfico 1. Variação da quantidade produzida e da capacidade estática de armazenamento de grãos em Minas Gerais de 1980 a 20177.

(mil toneladas)

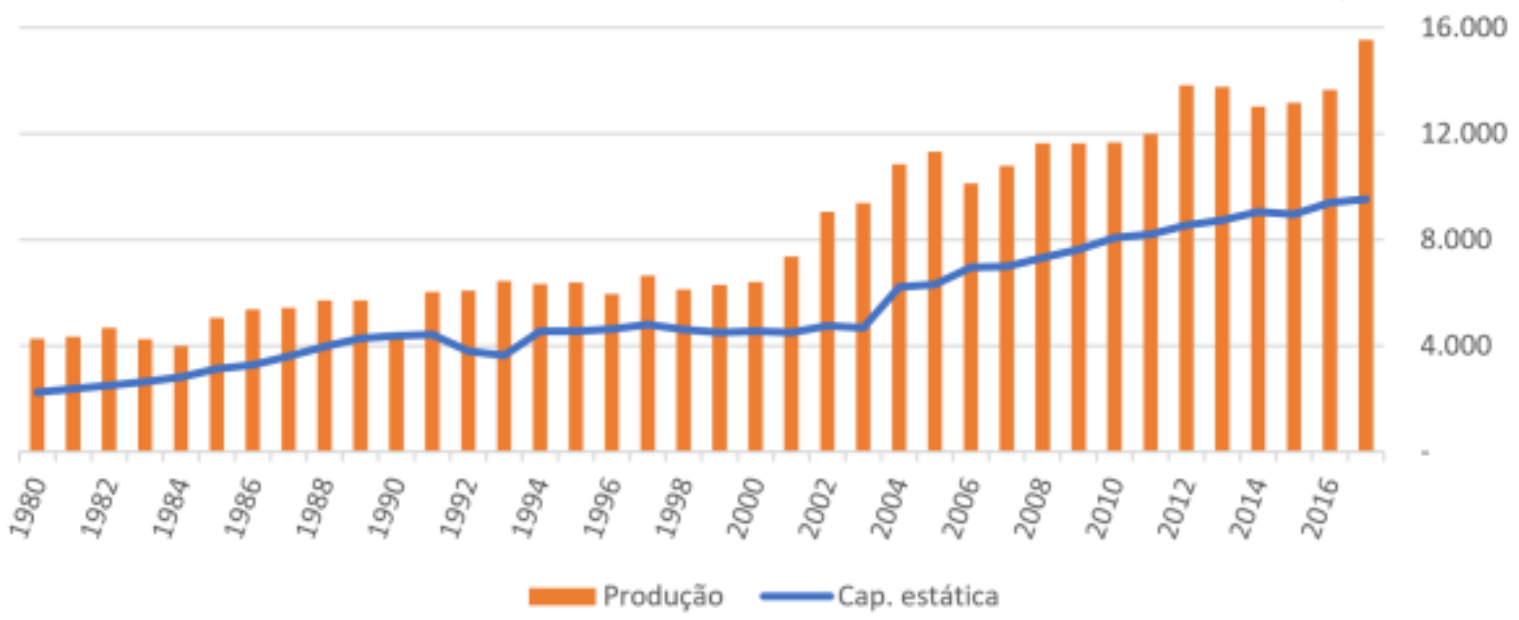

Fonte: Elaboração dos autores (Dados das séries históricas da CONAB)

\section{2- Materiais}

A base cartográfica consiste da carta topográfica na escala operacional de 1:250.000da malha de municípios do estado de Minas Gerais (853 no total), de acordo com a estrutura político-administrativa vigente em 01/07/2015 (IBGE, 2016b). Os dados utilizados são da Pesquisa Agrícola Municipal (PAM) de 2016, realizada pelo IBGE. A pesquisa é referente à produção agrícola do ano safra 15/16 no nível de agregação de municípios, obtidos na Tabela

\footnotetext{
${ }^{6}$ Dados não consolidados. Publicados na série "Indicadores CEI - Produto Interno Bruto - 40 Trimestre/2016", pela Fundação João Pinheiro (FJP) em 06 de abril de 2017, no site da instituição.

${ }^{7}$ Dados de 2017 não consolidados. Publicados em "Evolução da Capacidade Estática dos Armažéns" pela CONAB no site da instituição.
} 
5457 através do Sistema IBGE de Recuperação Automática (SIDRA). Foram considerados os dados de produção em toneladas de Algodão herbáceo (em caroço); Amendoim (em casca); Arroz (em casca); Aveia (em grão); Centeio (em grão); Cevada (em grão); Feijão (em grão); Girassol (em grão); Mamona (baga); Milho (em grão); Soja (em grão); Sorgo (em grão); Trigo (em grão); Triticale (em grão) e Café.

Dados de armazenagem foram obtidos no Sistema de Cadastro Nacional de Unidades Armazenadoras (SICARM) da Companhia Nacional de Abastecimento (CONAB) no nível da unidade armazenadora (1.259 no total) e foram agregados (capacidade estática e número de armazéns) ao nível de município. Importante informar que é de conhecimento dos autores que a base de dados de armazéns não possui todas as unidades existentes, pois a base não é construída de forma censitária. Faltando principalmente aquelas localizadas dentro das propriedades rurais. É necessário que o proprietário da unidade solicite a visita da CONAB e cadastro no sistema, mas mesmo assim é uma das bases pública mais completa que foi encontrada. A CONAB iniciou em setembro de 2017 um censo dos armazéns do país, que pretende atualizar as bases do Cadastro Nacional de Unidades Armazenadoras, mas o censo ainda está em curso. A Pesquisa de Estoques - IBGE também traz dados de capacidade estática útil das unidades armazenadoras nas Tabelas 259, 278, 911 e 5459. Entretanto, como houve divergência entre as bases de informação do IBGE e da CONAB, foi adotada a base da CONAB por apresentar maior nível de detalhamento.

Os softwares utilizados foram ESRI ArcGIS 10.4 (correção da base de dados,operações de geoprocessamento, análise espacial exploratória, regressão espacial e construção de cartogramas), R x64 3.4.1 (análise exploratória dos dados e análise estatística) e o Microsoft Excel 2016 (preparação e correção da base de dados e construção de gráficos).

\section{2- Métodos}

As bases foram organizadas e formatadas no programa Excel. As informações decapacidade estática e produção de grãos por município foram então reunidas em uma mesma tabela. Em seguida foi feita a junção com o shapefile de municípios, utilizando a ferramenta join do ArcGIS. De acordo com a recomendação da literatura (SNYDER, 1987; MITCHELL, 2005), o sistema de coordenadas da malha municipal de Minas foi previamente alterado para um sistema de coordenadas projetadas cônico equidistante, afim de preservar as distâncias e não interferir no cálculo da regressão GWR. O sistema escolhido foi o South AmericaEquidistantConic. 


\section{3- Análise exploratória dos dados}

Para a determinação da autocorrelação espacial da variável capacidade estática dearmazenagem foi feito o teste I de Moran, com o método de distância euclidiana, relação espacial fundamentada no inverso da distância, e limiar de pesquisa de vizinhança determinado pelo software para que cada ponto tenha pelo menos um vizinho.

Segundo Câmara et al. (2002), um aspecto fundamental na análise exploratóriaespacial é a caracterização da dependência espacial, que mostra como os valores estão correlacionados no espaço. As funções utilizadas para estimar quanto o valor observado de um atributo de uma região é dependente dos valores dessa mesma variável nas localizações vizinhas são a autocorrelação espacial e o variograma. Uma das formas de medir a autocorrelação espacial é através do índice I de Moran, cujo valor varia de -1 a +1 , e indica quanto cada área analisada é semelhante à sua vizinhança imediata.

Para completar a análise exploratória dos dados e identificação de parâmetros foifeita a regressão global OrdinaryLeastSquares (OLS), em português Mínimos Quadrados Ordinários (MQO). Esse modelo procura minimizar a soma dos quadrados dos resíduos, sendo um método direto, e que possui uma teoria bem desenvolvida. A aplicação dessa regressão ao conjunto de dados se dá para identificar o comportamento global dos dados, já que a análise de regressão realizada na sequência (GWR) é local. O modelo foi ajustado aos dados agregados a nível de município, nível da informação de produção (única variável explicativa do modelo).

\section{4- Modelo de Regressão}

A modelagem foi feita através da ferramenta GeographicallyWeightedRegression ${ }^{8}$ do software ArcGIS do conjunto de ferramentas de estatística espacial no sub-conjunto modelagem de relacionamentos espaciais. Foi definida como variável dependente a capacidade estática de armazenagem e como variável explicativa a produção. O tipo de Kernel foi definido como fixo. O método de largura de banda foi definido como AICc (Critério de Informação de Akaike corrigido) para que a ferramenta encontre a distância ideal ou o número de vizinhos automaticamente. Não foram utilizados pesos e todas as outras configurações de ambiente foram mantidas como default.

Os resultados da GWR foram analisados pela Soma dos Quadrados dos Resíduos,e pelo coeficiente de determinação $\left(\mathrm{R}^{2}\right)$ global e local. Especial atenção foi dada a análise dos resíduos

\footnotetext{
${ }^{8}$ A ideia da GWR é estimar os parâmetros do modelo de regressão para cada ponto observado doconjunto de dados, utilizando as demais observações ponderadas pela distância a este ponto.
} 
padronizados para identificar os municípios com excedente e déficit de armazenagem, em relação a capacidade estática prevista pela regressão em função da produção de grãos local.

\section{4- RESULTADOS E DISCUSSÃO}

\section{1- Análises exploratórias}

Tanto a nível da unidade armazenadora, quanto a nível de município, os dados decapacidade estática de armazenamento apresentaram autocorrelação espacial, com um padrão de agrupamento espacial (Tabela 1).

Tabela 1. Resumo da estatística I de Moran global para a capacidade estática de armazenamento.

\begin{tabular}{ccc}
\hline Indicador $\backslash$ Nível dos dados & Pontual & Agregado a nível de município \\
\hline Índice de Moran & 0,122324 & 0,041101 \\
Índice esperado & $-0,000795$ & $-0,001174$ \\
Variância & 0,002754 & 0,000065 \\
z-score & 2,346037 & 5,228092 \\
Valor-p & 0,018974 & 0 \\
Dist. limite de pesquisa de vizinhança $(\mathrm{km})$ & 107,196 & 66,553 \\
\hline
\end{tabular}

Para a regressão OLS, o teste Konker (BP) foi 45,849 Prob $\left(>X^{2}\right)$, indicando que arelação modelada não é consistente, devido a não-estacionariedade ou heterocedasticidade. A Estatística Joint Wald indicou significância global do modelo. O AICc para o modelo é 20.956,92. $\mathrm{O} \mathrm{R}^{2}$ foi de 0,367 e o $\mathrm{R}^{2}$ ajustado 0,366 , um ajuste relativamente baixo. $\mathrm{O}$ teste Jarque-Bera foi estatisticamente significativo apontando que as predições do modelo estão enviesadas, pois os resíduos não são normalmente distribuídos.

Portanto não foram atendidos os pressupostos do método OLS. Devido aodiagnóstico inicial dos resultados, foi feito um I de Moran para checar se os resíduos não são espacialmente autocorrelacionados. Obtendo I de Moran: 0,009277; variância: 0,000054; zscore: 1,419715 e valor-p: 0,155691, onde o padrão não parece ser significativamente diferente da distribuição por chance aleatória. 


\section{2- Análise de regressão espacial local - GWR}

Os resultados da Regressão Geograficamente Ponderada são apresentados na Tabela 2. O $\mathrm{R}^{2}$ local de ajuste do modelo pode ser observado na Figura 2. O $\mathrm{R}^{2}$ global foi de 0,687 , enquanto o $\mathrm{R}^{2}$ local variou de 0,00 até 0,99 . O modelo apresentou um ajuste razoavelmente bom, e analisando o padrão dos resíduos do modelo (Figura 3), podemos inferir que a incorporação de mais variáveis explicativas, como portos secos, plantas industriais de processamento e polos comerciais pode melhorar a explicação deste. A lógica da inserção dessas novas variáveis se sustenta na premissa que os armazéns são necessários tanto onde os grãos são produzidos quanto onde são consumidos, equalizando assim a logística na cadeia.

Tabela 2. Resumo da estatística da regressão GWR.

\begin{tabular}{lr}
\hline \multicolumn{1}{c}{ Variável } & Valor \\
\hline Largura de banda $(\mathrm{m})$ & $66.546,5179$ \\
Soma dos Quadrados dos Resíduos & $1,1475 \times 10^{12}$ \\
Sigma & $39.004,1035$ \\
AICc & $20.505,4496$ \\
$\mathrm{R}^{2}$ & 0,6870 \\
$\mathrm{R}^{2}$ Ajustado & 0,6464 \\
\hline
\end{tabular}

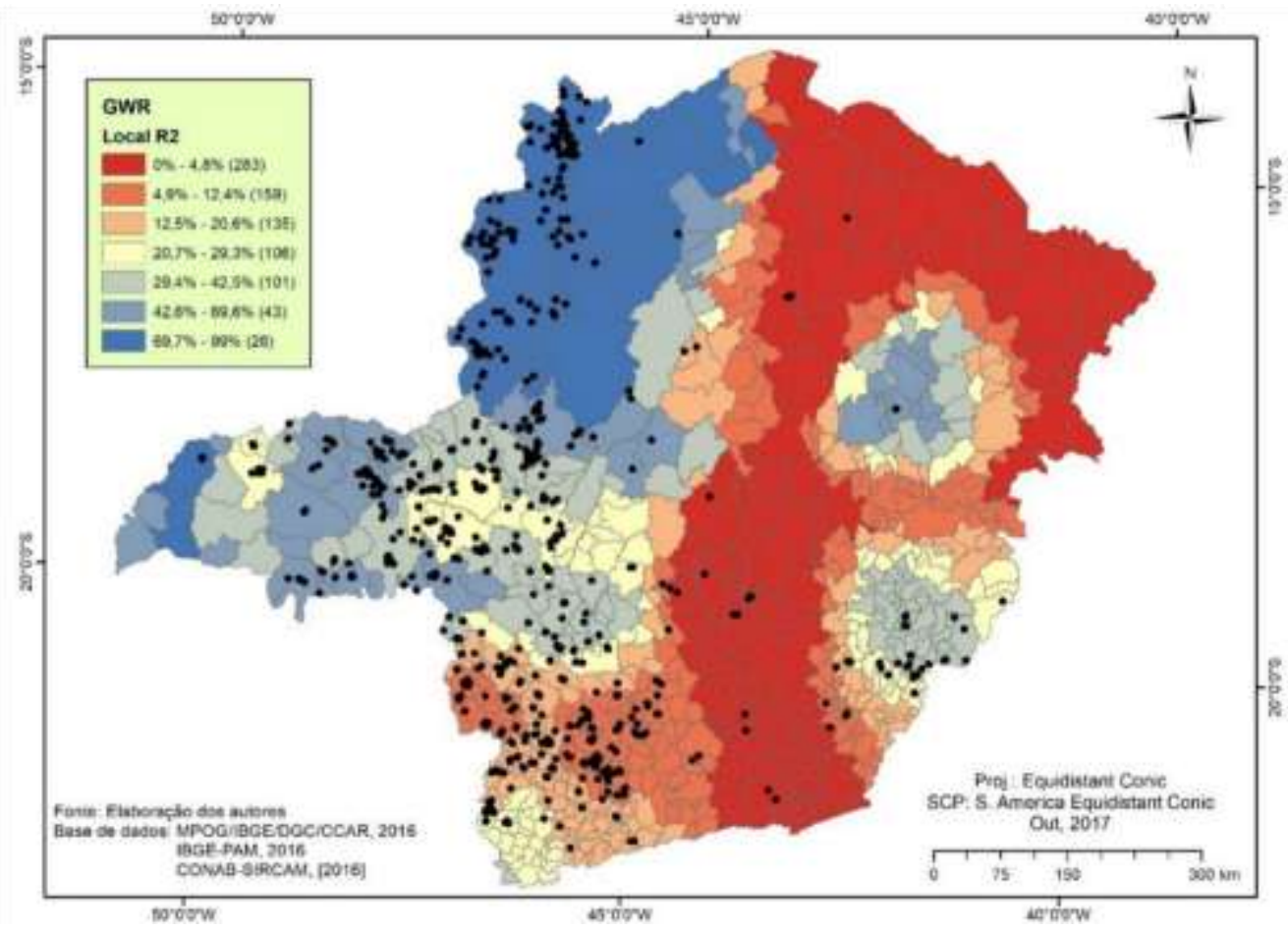

Figura 2. $R^{2}$ local da GWR da capacidade estática em função da produção. 
A regressão não apresentou problemas de multicolinearidade local, com os valoresdessa condição variando de 1,274 a 3,019 para todos os municípios. A Soma dos Quadrados dos Resíduos (Residual Squares) apresentou um valor alto, indicando grande dispersão dos dados. O valor de Sigma assim como a Soma dos Quadrados dos Resíduos, também apresentou um valor alto. Assim como na regressão OLS não há uma formação de clusters bem definida nos resíduos da GWR. O I de Moran foi de -0,017923 para os resíduos padronizados, com índice esperado de -0,001174; variância: 0,000069; z-score: -2,022143 e valor-p: 0,043162, indicando um padrão de dispersão fraca dos resíduos.

Com exceção da região Sul do estado, as áreas que apresentam produção e unidadesarmazenadoras foram as que obtiveram melhor ajuste do modelo $\left(\mathrm{R}^{2}\right.$ local). O que pode indicar que se excluídos os municípios que tem um baixo nível de produção e não possuem unidades armazenadoras o modelo pode melhorar.

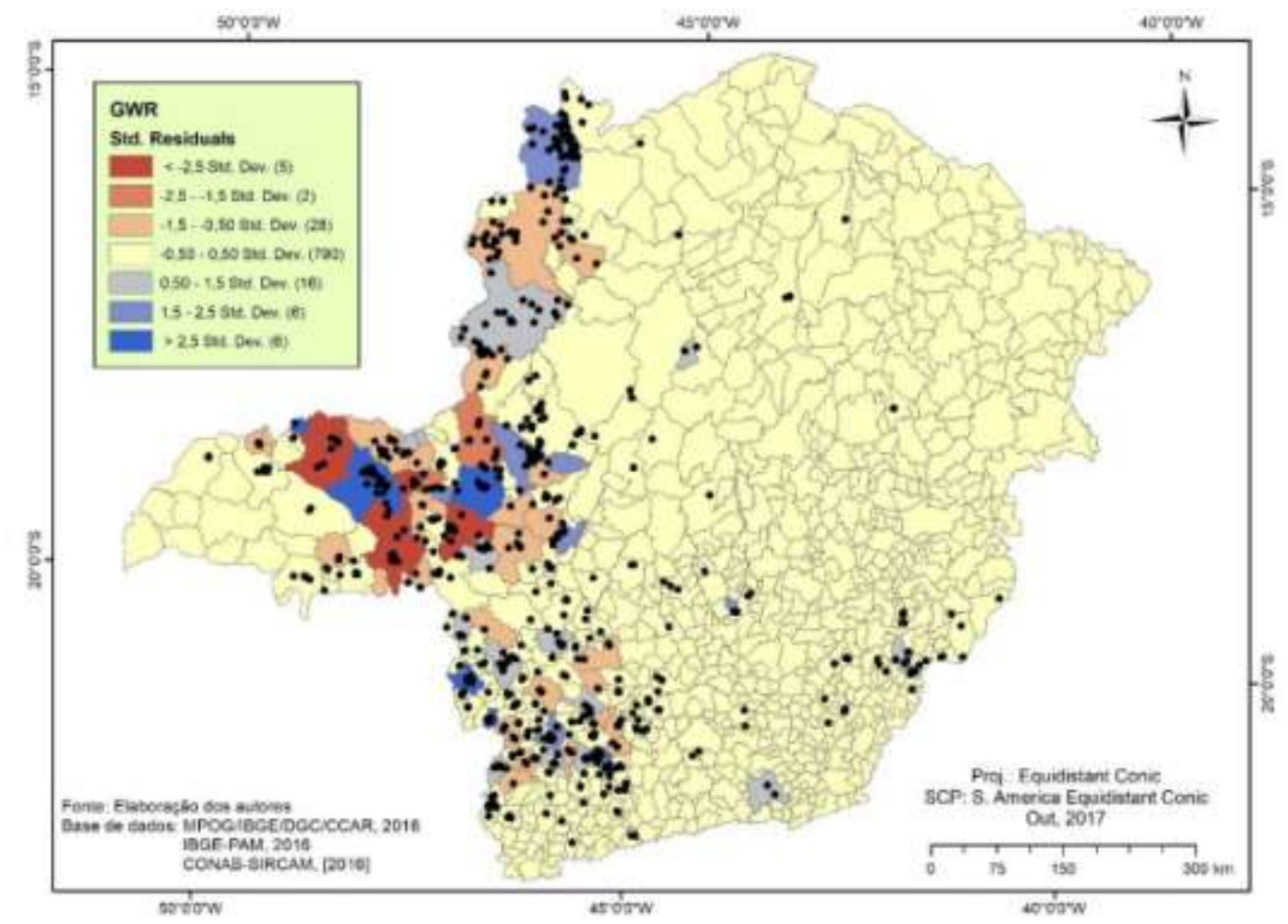

Figura 3. Resíduos padronizados (Standardizeed Residuah) da GWR da capacidade estática pela produção.

$\mathrm{Na}$ Figura 3 podemos observar o mapa com os resíduos padronizados obtidos nomodelo GWR. A maioria dos municípios (790) apresentaram resíduo padronizado entre -0,5 e 0,5 desvio padrão (DP). Ou seja, o modelo se aproximou das observações da maior parte dos dados. Sendo que 6 municípios (Uberlândia, Varginha, Patrocínio, Guaxupé, São Sebastião do Paraíso e 
Araporã) apresentam resíduo acima de 2,5 DP, indicando capacidade estática de armazenamento acima do esperado para a sua produção. Outras 5 municipalidades (Uberaba, Monte Alegre de Minas, Tupaciguara, Perdizes e Indianópolis) precisam de atenção, pois apresentaram desvio negativo além de -2,5 DP. Ou seja, apresentam déficit significativo.

As cidades com excedente de capacidade estática são importantes polos dedistribuição e comercialização de commodities agrícolas. Uberlândia é um polo do agronegócio que abriga fábricas de processamento de grãos, empresas de sementes, de fertilizantes, etc. Além de ser um centro logístico com transbordos que fazem a mudança do modal de transporte rodoviário para o ferroviário dos grãos destinados à exportação, produzidos principalmente nos estados de Minas Gerais, Mato Grosso e Goiás. Varginha é um dos principais centros de comércio e produção de café do Brasil e do mundo, sendo referência na produção cafés de alta qualidade. Patrocínio é também um grande produtor agrícola, com destaque para o café, e devido a sua localização recebe a produção de municípios vizinhos. Em Guaxupé a produção de café é tão importante, que a cidade é a sede de uma das maiores cooperativas da América Latina, a Cooxupe. São Sebastião do Paraíso é um médio produtor, com o principal produto sendo o café. Mas devido à sua localização, na divisa de Minas e São Paulo, e com importantes rodovias cruzando o município, tem capacidade estática superior à produção. Por último, Araporã se apresenta como exceção entre as cidades com excedente de armazenagem. Possui grande desvio por apresentar uma produção agrícola muito pequena e dois armazéns, também com capacidade pequena, mas bem superior à produção agrícola da localidade, portanto, devem armazenar a produção de municípios vizinhos.

Já as cidades com capacidade estática bem abaixo do esperado situam-se no Triangulo Mineiro, no entorno de Uberlândia. Nesse caso a produção vai direto da lavoura para a exportação, ou para as unidades de processamento.

\section{5- CONCLUSÃO}

A relação entre produção e armazenamento de grãos foi modelada e analisada. Nãoforam encontrados grandes desvios negativos que mereçam especial atenção. Em geral a maioria das municipalidades apresentam déficit de armazenamento, porém pequeno. Quando o déficit é significativo, os municípios situam-se próximos de outros que possuem excedente.

Vale lembrar que o modelo de regressão ajusta a equação baseado nos dados deprodução agrícola e armazenagem observados. Como a maioria dos municípios possui algum nível de 
déficit, isso é assumido como natural e incorporado ao modelo. O que não quer dizer que a situação seja confortável e não exista um déficit de capacidade estática no estado. Dos 853 municípios estudados, 785 apresentam algum nível de déficit de armazenagem. A análise proposta serve para identificar grandes desvios dentre os municípios mineiros, o que praticamente não foi observado, e quando foi era perfeitamente justificável.

Um ponto importante de ser mencionado é que a capacidade estática dearmazenamento não deveria ser adotada neste tipo de análise. Pela experiência prática dos autores foi observado que existe grande diferença do giro (quantas vezes o armazém enche em um ano) entre os tipos de armazém. Por isso a capacidade estática deve ponderada por um fator do tipo de propriedade do armazém. Além disso, as safras não coincidem e não se colhe toda a produção simultaneamente.

Novos estudos devem ser realizados com a incorporação de portos secos, plantasindustriais de processamento e polos comerciais como variáveis explicativas do modelo. Com a ponderação do giro de estoque de acordo com o tipo de armazém. Considerando a recomendação de capacidade estática da $\mathrm{FAO}$ de 1,2 vezes a produção, e avaliando a possibilidade de exclusão de áreas do estado no modelo.

Estudos dessa natureza, identificando as características das regiões com capacidadede armazenamento deficitária, devem ser utilizados para guiar políticas públicas e projetos de investimento, pois o posicionamento adequado de novas instalações reduz custos e agrega valor na cadeia logística.

A situação da armazenagem é de tamanha relevância que o Plano Agrícola e Pecuário (PAP) 2017/2018 publicado pelo governo federal brasileiro, definiu a expansão da capacidade de armazenagem e inovação tecnológica como prioridades do plano. Direcionando recursos estatais e estabelecendo a menor taxa de juros dentre todas as áreas atendidas com financiamento pelo PAP 2017/18 (MAPA, 2017b).

\section{REFERÊNCIAS BIBLIOGRÁFICAS}

ALBUQUERQUE, P.H.M.; MEDINA, F.A.S.; SILVA, A.R. Regressão Logística Geograficamente Ponderada Aplicada a Modelos de CreditScoring. R. Cont. Fin, v. 28, n. 73, p. 93-112, 2017.

BIAGI, J. D.; BERTOL, R.; CARNEIRO, M. C. Armazéns em Unidades centrais de Armazenamento. In: LORINI, I.; MIIKE, L. H.; SCUSSEL, V.M. (Ed.). Armazenagem de Grãos. Campinas: IBG, 2002. Cap. 3.3; p. 157-161. 
BRUNSDON, C.; FOTHERINGHAM, A. S.; CHARLTON, M. E. Geographically weighted regression: a method for exploring spatial nonstationarity. GeographicalAnalysis, v. 28, n. 4, p. 281-298, 1996.

CÂMARA, G.; CARVALHO, M.S.; CRUZ, O.G.; CORREA, V. Análise Espacial de Áreas. In: FUKS, S.D.; CARVALHO, M.S.; CÂMARA, G.; MONTEIRO, A.M.V. (Ed.) Análise Espacial de Dados Geográficos. São José dos Campos: DPI - INPE, 2002. Disponível em: http://www.dpi.inpe.br/gilberto /livro/analise/cap5-areas.pdf. Acesso em: 21 nov. 2017

CARVAlho, L. E. X.; SILVA, H. N.; LOUREIRO, C. F. G.; MENESES, H. B. Regressão Linear Geograficamente Ponderada em ambiente SIG. Transportes, v. 14, n. 2, p. 18-26, 2006.

Centro de Estudos Avançados em Economia Aplicada (CEPEA). Esalq, USP (Comp.). PIB do Agronegócio: Dados de 1996 a 2017. 2017. Parceria CEPEA - CNA. Disponível em: https:// www.cepea.esalq.usp.br/br/pib-do-agronegocio-brasileiro.aspx. Acesso em: 28 nov. 2017.

Companhia Nacional de Abastecimento (CONAB). Cadastro Nacional de Unidades Armazenadoras. $2016 . \quad$ Disponível em: http://consultaweb.conab.gov.br/consultas/consultaArmazem.do?method=acao CarregarConsulta. Acesso em: 18 out. 2017.

Monitoramento Agrícola Safra 2016/17: 4º levantamento. Acomp. safra bras. grãos, v. 4, n. 4, Brasília, 2017a. 160 p. Disponível em: http://www.conab.gov.br/conteud os.php?a $=1253 \&$ ordem $=\mathrm{M} \%$ EAs $/$ Ano $\% 20 \mathrm{da} \% 20$ publica $\%$ E7\%E3o. Acesso em: 20 nov. 2017.

Evolução da Capacidade Estática dos Armazéns. Brasília, 2017b. 7 p. Disponível em: http://www.conab.gov.br/conteudos.php?a=1077\&t=. Acesso em: 21 nov. 2017.

Food and Agriculture Organization of the United Nations (FAO). Price volatility in food and agricultural markets: policy responses. Relatório de Políticas ao G20. p. 68, 2011. Disponível em: http://faostat.fao.org. Acesso em: 10 nov. 2017.

Fund. João Pinheiro (FJP). Produto Interno Bruto (PIB) de Minas Gerais: Relatório Anual 2015. Fundação João Pinheiro, Diretoria de Estatística e Informações. Belo Horizonte: FJP, 2017a.

PIB Trimestral de Minas Gerais: $4^{\circ}$ trimestre de 2016. Indicadores CEI. Fundação João Pinheiro, Centro de Estatística e Informações. Belo Horizonte: FJP, 2017b.

GILBERT, A.; CHAKRABORTY, J. Using geographically weighted regression for environmental justice analysis: Cumulative cancer risks from air toxics in Florida. Social Science Research, v. 40, n. 1, p. 273-286, 2011.

HUANG, Y.; LEUNG, Y. Analyzing regional industrialization in Jiangsu province using geographically weighted regression. JournalofGeographical Systems, v.4, n.2, p. 233-249, 2002.

Instituto Brasileiro de Geografia e Estatística (IBGE). Pesquisa Agrícola Municipal. 2016a. Disponível em: https://sidra.ibge.gov.br/pesquisa/pam/tabelas. Acesso em: 18 out. 2017. 
Mapas: Malhas digitais. 2016b. Nota técnica. Disponível em: ftp://geoft p.ibge.gov.br/organizacao_do_territorio/malhas_territoriais/malhas_municipais/municipio_20 15/. Acesso em: 14 out. 2017.

Área Territorial Oficial: Consulta por Unidade da Federação. 2016c. Disponível em: https://www.ibge.gov.br/home/geociencias/cartografia/default_territ_area.shtm. Acesso em: 18 nov. 2017

Estimativas da população residente no Brasil e unidades da federação com data de referência em $1^{\circ}$ de julho de 2017. 2017. Disponível em: https://biblioteca.ibge.gov.br/ visualizacao/livros/liv100923.pdf. Acesso em: 18 nov. 2017.

LOBO, C.; FONSECA, D. F.; GARCIA, R. A. Verticalização e permeabilização do solo urbano nos setores censitários de Belo Horizonte/MG. Rev. Bras. Estud. Urbanos Reg., v.17, n.3, p.215228, Recife, set./dez. 2015.

MENDONÇA, A. C. Desenvolvimento de um modelo de previsão da demanda de passageiros do transporte rodoviário interestadual utilizando regressão com efeitos espaciais locais. 2008. 123 f. Dissertação (Mestrado em Transportes) - Universidade de Brasília, Brasília, 2008.

Ministério da Agricultura, Pecuária e Abastecimento (MAPA). Secretaria de Política Agrícola (SPA). VPB - Valor Bruto da Produção: Outubro 2017. 2017a. Disponível em: http://www.agricultura.gov.br/ noticias/valor-da-producao-de-2017-e-de-r-533-5-bilhoes.

Acesso em: 21 nov. 2017.

Plano Agrícola e Pecuário 2017/2018. 2017b. Disponível em: http://www.agric ultura.gov.br/assuntos/sustentabilidade/plano-agricola-e-pecuario. Acesso em: 20 nov. 2017.

MITCHELL, A. The ESRI guide to GIS analysis: Spatial measurements and statistics. v. 2. Redlands: ESRI Press. 2005.

OVERMARS, K.P.; KONING, G.H.J.; VELDKAMP, A. Spatial autocorrelation in multi-scale land use models. Ecological Modelling, v.164, p.257-270, 2003.

PATINO, M. T. O.; MACHADO, M. F.; NASCIMENTO, G. T.; ALCANTARA, M. R. Analysis and forecast of the storage needs of soybeans in Brazil. Eng. Agríc. [online]. 2013, vol.33, n.4, p.834-843.. Disponível em: http://dx.doi.org/10.1590/S0100-69162013000400022. Acesso em: 27 nov. 2017.

SEE, L.; SCHEPASCHENKO, D.; LESIV, M.; MCCALLUM, I.; FRITZ, S.; COMBER, A; OBERSTEINER, M. Building a hybrid land cover map with crowdsourcing and geographically weighted regression. ISPRS J. of Photogrammetry and Remote Sensing. v. 103, p. 48-56, 2015.

SHARMA, V.; IRMAK, A.; KABENGE, I.; IRMAK, S. Application of GIS and geographically weighted regression to evaluate the spatial nonstationarity relationships between precipitation vs. irrigated and rainfed maize and soybean yields. Transactions of the ASABE. v. 54, n. 3, p. 953972, 2011. doi: 10.13031/2013.41227 
SNYDER, J. Map Projections: A Working Manual. Report.Professional Paper Series. Washington: USGS Publications Warehouse, 1987. Series number: 1935. 385 p.

SOARES-FILHO, B. S.; GARCIA, R. A.; RODRIGUES, H.; MORO, S.; NEPSTAD, D. Nexos entre as dimensões socioeconômicas e o desmatamento: a caminho de um modelo integrado. In: BATISTELLA, M.; ALVES, D. S.; MORAN, E. (Org.). Amazônia. Natureza e Sociedade em Transformação. São Paulo: Edusp, 2008. 